Louisiana State University

LSU Digital Commons

$1-1-2019$

\title{
Naringenin Promotes Thermogenic Gene Expression in Human White Adipose Tissue
}

Candida J. Rebello

Pennington Biomedical Research Center

Frank L. Greenway

Pennington Biomedical Research Center

Frank H. Lau

LSU Health Sciences Center - New Orleans

Yuan Lin

LSU Health Sciences Center - New Orleans

Jacqueline M. Stephens

Pennington Biomedical Research Center

See next page for additional authors

Follow this and additional works at: https://digitalcommons.Isu.edu/biosci_pubs

\section{Recommended Citation}

Rebello, C., Greenway, F., Lau, F., Lin, Y., Stephens, J., Johnson, W., \& Coulter, A. (2019). Naringenin Promotes Thermogenic Gene Expression in Human White Adipose Tissue. Obesity, 27 (1), 103-111. https://doi.org/10.1002/oby.22352 
Authors

Candida J. Rebello, Frank L. Greenway, Frank H. Lau, Yuan Lin, Jacqueline M. Stephens, William D. Johnson, and Ann A. Coulter 


\title{
Naringenin Promotes Thermogenic Gene Expression in Human White Adipose Tissue
}

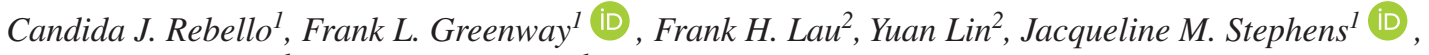 \\ William D. Johnson ${ }^{l}$, and Ann A. Coulter ${ }^{l}$
}

Objective: Naringenin, a citrus flavonoid, prevents diet-induced weight gain and improves glucose and lipid metabolism in rodents. There is evidence that naringenin activates brown fat and increases energy expenditure in mice, but little is known about its effects in humans. The goal of this study was to examine the effects of naringenin on energy expenditure in adipose tissue.

Methods: Human white adipocyte cultures (hADSC) and abdominal subcutaneous adipose tissue (pWAT) were treated with naringenin for 7 to 14 days. Expression (quantitative real-time polymerase chain reaction, immunoblotting) of candidate genes involved in thermogenesis and glucose metabolism was measured. Oxygen consumption rate was measured in hADSC using a Seahorse flux analyzer.

Results: In hADSC, naringenin increased expression of the genes associated with thermogenesis and fat oxidation, including uncoupling protein 1 and adipose triglyceride lipase, and key factors associated with insulin sensitivity, including glucose transporter type 4, adiponectin, and carbohydrate-responsive elementbinding protein $(P<0.01)$. Similar responses were observed in pWAT. Basal, ATP-linked, maximal and reserve oxygen consumption rate increased in the naringenin-treated hADSC $(P<0.01)$.

Conclusions: Naringenin increases energy expenditure in hADSC and stimulates expression of key enzymes involved in thermogenesis and insulin sensitivity in hADSC and pWAT. Naringenin may promote conversion of human white adipose tissue to a brown/beige phenotype.

Obesity (2019) 27, 103-111. doi:10.1002/oby.22352

\section{Introduction}

Browning of white adipose tissue has garnered interest in research on obesity because it engenders adaptive thermogenesis, a process mediated by mitochondrial uncoupling protein 1 (UCP1). When activated, UCP1 short-circuits the electrochemical gradient that drives ATP synthesis. Downregulation of the proton gradient stimulates respiratory chain activity and provokes heat production through combustion of available substrates (1). This type of metabolically active and energy-dissipating tissue offers a potential solution to counteract excessive fat accumulation, especially because brown adipose tissue is typically low in humans and inversely correlates with BMI (2). The browning of adipose tissue may also modulate systemic insulin action through nonthermogenic mechanisms such as the secretion of insulin-sensitizing factors from adipose tissue (1). Thus, the therapeutic potential of brown fat transcends obesity and may be considered for other metabolic abnormalities such as insulin resistance.

Carbohydrate-responsive element-binding protein (ChREBP) is a transcription factor that is known to be expressed as $\alpha$ and $\beta$ alternatively spliced isoforms. In humans, expression of both isoforms in white adipose tissue correlates with insulin sensitivity (3-5); however, obesity is associated with a specific reduction in ChREBP $\beta$ expression $(3,5)$. Overexpression of ChREBP in mice fed a Western diet was shown to enhance systemic insulin sensitivity, reduce fat mass, and increase expression of the genes associated with thermogenesis, suggesting a role for ChREBP in whole-body insulin sensitivity and brown adipose tissue function (6). To date, the only compound known to stimulate ChREBP expression (in mouse adipose tissue) is the incretin mimetic liraglutide (7).

\footnotetext{
${ }^{1}$ Pennington Biomedical Research Center, Louisiana State University System, Baton Rouge, Louisiana, USA. Correspondence: Frank L. Greenway (frank. greenway@pbrc.edu) ${ }^{2}$ Louisiana State University Health Sciences Center, Louisiana State University, New Orleans, Louisiana, USA.

Funding agencies: This publication was supported in part by the National Center For Complementary and Integrative Health and the Office of Dietary Supplements of the National Institutes of Health (NIH) under award number P50AT002776, which funds the Botanical Dietary Supplements Research Center of Pennington Biomedical Research Center, in part on work that was supported by NIH under award number T32 A T004094 and in part by 1 U54 GM104940 from the National Institute of General Medical Sciences of $\mathrm{NIH}$, which funds the Louisiana Clinical and Translational Science Center. This work utilized the facilities of the Genomics Core that are supported in part by Centers of Biomedical Research Excellence (NIH8 1P30GM118430-01) and Nutrition Obesity Research Center (P30DK072476) grants from NIH.
}

Disclosure: The authors declared no conflict of interest.

Received: 1 June 2018; Accepted: 1 October 2018; Published online 2 December 2018. doi:10.1002/oby.22352 
Strategies to enhance adipose tissue glucose uptake and restore depleted levels of ChREBP expression can be particularly beneficial to individuals with obesity and insulin resistance. Naringenin, a citrus flavonoid, reduces diet-induced weight gain, increases energy expenditure, and improves glucose and lipid metabolism in animal models (8-12). Activation of AMP-activated protein kinase (AMPK), regulation of insulin signaling pathways and lipid metabolism, relief from oxidative stress in pancreatic beta cells, and increases in brown fat thermogenic gene expression are among the mechanisms that have been implicated (8-11). The effects of naringenin on energy expenditure and expression of the genes involved in glucose metabolism in human adipose tissue have not been investigated.

The literature is replete with the beneficial effects of naringenin in obesity, cardiovascular disease, and cancer (13). However, grapefruit has been shown to increase the bioavailability of drugs administered orally. Several compounds in grapefruit including the flavonoids (naringin and naringenin), furanocoumarins, and sesquiterpenes have been implicated (14). Naringenin, being polyphenolic and high in electrons, can theoretically inhibit cytochrome P450 enzymes and enhance the bioavailability of medications including statins. Although inhibition of the cytochrome P450 enzyme system has been demonstrated in vitro and in rodent models, the results of in vivo studies in humans suggest that naringenin is not the main inhibitory compound in grapefruit $(15,16)$. The most potent inhibitor of CYP3A4, the main enzyme in the cytochrome P450 family that is involved in drug metabolism, is bergapten, a furanocoumarin derivative (17).

We investigated the effects of naringenin on energy expenditure in human adipocytes and primary human white adipose tissue. Preliminary studies demonstrated that exposure of human adipocytes to naringenin upregulated the mRNA expression of UCP1 and glucose transporter type 4 (GLUT4, also known as solute carrier family 2 member 4 [SLC2A4]). We therefore hypothesized that naringenin acts in white adipose tissue to promote conversion of human white adipocytes to the beige/brown phenotype.

\section{Methods}

\section{Materials}

Hyclone DMEM/Ham's F12 (DMEM/F12 1:1) and Gibco fetal bovine serum (FBS) were purchased from Thermo Fisher Scientific (Waltham, Massachusetts). Rosiglitazone was purchased from AK Scientific (Union City, California). All other reagents used in the medium were purchased from Sigma-Aldrich (St. Louis, Missouri). Naringenin (purity $\geq 98 \%$ ) was purchased from Cayman Chemicals (Ann Arbor, Michigan). Protease and phosphatase were purchased from Cell Signaling Technology (CST; Danvers, Massachusetts), radioimmunoprecipitation assay buffer was purchased from Sigma-Aldrich, and TGX protein gels were purchased from Bio-Rad (Hercules, California). Seahorse XF assay medium and pyruvate solution were purchased from Agilent (Santa Clara, California). Carbonyl cyanide-4-(trifluoromethoxy) phenylhydrazone (FCCP), oligomycin, and antimycin A were purchased from Sigma-Aldrich.

\section{Cell culture (adipocytes)}

Human adipose-derived stem cells from female donors with overweight or obesity were purchased from LaCell, LLC (New Orleans, Louisiana). Preadipocytes were seeded in DMEM/F12 1:1 supplemented with $10 \%$ FBS and 1\% antibiotic (penicillin/streptomycin/ amphotericin). One day after reaching confluence, cells were differentiated (human white adipocyte cultures [hADSC]) in media containing 70\% DMEM and 30\% DMEM/F12 1:1 supplemented with 3\% FBS, $1 \%$ antibiotic, $1 \mu \mathrm{M}$ dexamethasone, $33 \mu \mathrm{M}$ biotin, $0.1 \mu \mathrm{M}$ insulin, $20 \mu \mathrm{M}$ pantothenate, $5 \mu \mathrm{M}$ rosiglitazone, and $500 \mu \mathrm{M}$ 3-isobutylmethylxanthine. After induction in the differentiation media (4 or 5 days), cells were maintained in medium composed of $70 \%$ DMEM and $30 \%$ DMEM/F12 1:1 supplemented with 3\% FBS, $1 \mu \mathrm{M}$ dexamethasone, $33 \mu \mathrm{M}$ biotin, $0.1 \mu \mathrm{M}$ insulin, and $20 \mu \mathrm{M}$ pantothenate (day 0 ). Cells were kept at $37^{\circ} \mathrm{C}$ in a humidified atmosphere of $95 \%$ air and $5 \% \mathrm{CO}_{2}$. Treatments $[8 \mu \mathrm{M}$ naringenin based on pharmacokinetic study done in humans (18)] started from day 3 were administered every 2 days over a period of 6 days, and cells were harvested the following day (day 10). Dexamethasone was removed from the maintenance medium during treatment to promote browning of adipocytes (19), and FBS was inactivated by heating for 30 minutes at $56^{\circ} \mathrm{C}$. Following harvest, TRIzol reagent (Sigma-Aldrich) was added to lyse adipocytes, and the contents of each well were homogenized. RNeasy Mini Kit (Qiagen, Germantown, Maryland) was used to isolate RNA following manufacturer's protocol.

\section{Ex vivo adipose tissue explants}

Abdominal subcutaneous adipose tissue (pWAT) procured from four females, subjects $1,2,3$, and 4 undergoing elective panniculectomies, was mechanically minced into 0.5 - to $1-\mathrm{mm}$ diameter segments and $500 \mu \mathrm{L}$ was suspended in $3 \mathrm{~mL}$ of adipocyte maintenance medium for each replicate (day 0$)$. Treatments $(8 \mu \mathrm{M}$ naringenin) started from day 3 were administered to subjects 1,2 , and 3 every 2 days over a period of 14 days or were started at day 10 and treated over 7 days. All pWAT samples, including those from the untreated controls, were harvested on the same day. In a separate experiment, pWAT from subjects 1, 2, and 4 was treated in a similar fashion with GW $7647(0.3 \mu \mathrm{M})$. At the end of the treatment period, adipocytes were isolated from pWAT after standard collagenase digestion, and RNA was extracted with TRIzol. Subject characteristics are provided in Table 1.

\section{Quantitative real-time polymerase chain reaction}

The RNA was quantified using Invitrogen Qubit RNA Broad-Range Assay Kit and read using a Qubit Fluorometer (Thermo Fisher Scientific). Reverse transcriptase and real-time polymerase chain reaction (RT-PCR) were conducted in one reaction with the reverse PCR primer priming complementary DNA (cDNA) synthesis using SuperScript III Platinum One-Step qRT-PCR Kit with ROX from Invitrogen (Thermo Fisher Scientific). Amplicons were designed to span intron-exon junctions to avoid amplification of genomic sequences. Primer and probe oligonucleotide sets were purchased from Integrated DNA Technologies (Coralville, Iowa) and are provided in Table 2. RPL13A (TaqMan identifier Hs04194366_g1, Thermo Fisher

TABLE 1 Characteristics of donors of adipose tissue used in explant experiments

\begin{tabular}{llll}
\hline Subject & Age & BMI & Race \\
\hline $\mathbf{1}$ & 33 & 29.2 & Caucasian \\
$\mathbf{2}$ & 45 & 25.8 & Caucasian \\
$\mathbf{3}$ & 39 & 29.3 & African American \\
$\mathbf{4}$ & 35 & 26.1 & African American \\
\hline
\end{tabular}


TABLE 2 Primer and probe sequences for qPCR

\begin{tabular}{|c|c|c|c|}
\hline Gene & Primer and probe & Sequences & $\begin{array}{c}\mathrm{NCBI} \text { reference } \\
\text { sequence }\end{array}$ \\
\hline \multirow[t]{3}{*}{ Adiponectin } & Forward & CTGGTGAGAAGGGTGAGAAAG & NM_004797.2 \\
\hline & Reverse & сTCCTTTCCTGCCTTGGATT & \\
\hline & Probe & TGGTCCTAAGGGAGACATCGGTGA & \\
\hline \multirow[t]{3}{*}{ ATGL } & Forward & CGTGTACTGTGGGCTCATC & NM_020376.3 \\
\hline & Reverse & GGACACTGTGATGGTGTTCTTA & \\
\hline & Probe & ATGGTGGCATTTCAGACAACCTGC & \\
\hline \multirow[t]{3}{*}{ CPT1B } & Forward & TACCATGGGTGGATGTTTGAG & NM_013261.3 \\
\hline & Reverse & GTCTGGAAGCTGTAGAGCATAG & \\
\hline & Probe & TCTGGGCTATGTGTATCCGCCTTCTA & \\
\hline \multirow[t]{3}{*}{ ChREBP } & Forward & TCTGGACACAGCCGTCT & NM_032951 \\
\hline & Reverse & GCTTGGGGTCTTCAGGAA & \\
\hline & Probe & ATCAGAACTCAGGAAGGCGCTGG & \\
\hline \multirow[t]{3}{*}{ ChREBP $\beta$} & Forward & GAGCGGATTCCAGGTGAG & BG616809.1 \\
\hline & Reverse & TTGTTCAGGCGGATCTTGT & \\
\hline & Probe & TCATCAGTGGCAAGCTGGTGTCTC & \\
\hline \multirow[t]{3}{*}{ GLUT4 } & Forward & GTATCATCTCTCAGTGGCTTGG & NM_001042 \\
\hline & Reverse & ATAGGAGGCAGCAGCATTG & \\
\hline & Probe & AAAGGGCCATGCTGGTCAACAATG & \\
\hline \multirow[t]{3}{*}{ PGC-1 $\alpha$} & Forward & CACCAAACCCACAGAGAACA & NM_00145134.1 \\
\hline & Reverse & GGGTCAGAGGAAGAGATAAAGTTG & \\
\hline & Probe & AAAGAAGTCCCACACACAGTCGCA & \\
\hline \multirow[t]{3}{*}{ PGC-1 $\beta$} & Forward & GAGGCGCTTTGAAGTGTTTG & NM_001172699 \\
\hline & Reverse & GAACACCGGTAGGTGATGAAG & \\
\hline & Probe & CAGCACCTCGCACTCCTCAATCTC & \\
\hline \multirow[t]{3}{*}{ PRDM16 } & Forward & TCGGAAATCAGAAACTTTATTGCC & NM_199454 \\
\hline & Reverse & TCCTGCTTCTCACTGGCTA & \\
\hline & Probe & TCTCTGTTCGCGTTGATGCTTGGT & \\
\hline \multirow[t]{3}{*}{ SIRT1 } & Forward & GTTTCATGATAGCAAGCGGTTC & NM_001142498 \\
\hline & Reverse & GTCATGGTTCCTTTGCAACAG & \\
\hline & Probe & CTCGATGTCCTAGGTGCCCAGC & \\
\hline \multirow[t]{3}{*}{ UCP1 } & Forward & GAGGAGTGGCAGTATTCATTGG & NM_021833 \\
\hline & Reverse & CCGTGTAGCGAGGTTTGATT & \\
\hline & Probe & TTCAAGCACAGAGCCATCTCCACG & \\
\hline
\end{tabular}

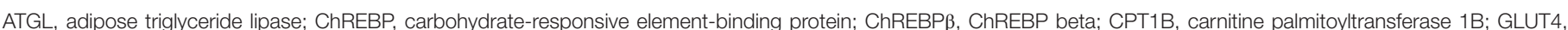

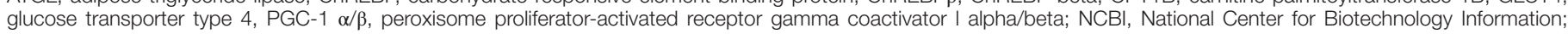
PRDM16, PR domain containing 16; qPCR, quantitative polymerase chain reaction; SIRT1, sirtuin 1; UCP1, uncoupling protein 1.

Scientific) was used as a housekeeping gene. The RT-PCR assay for each sample was conducted in duplicate using the protocol for Applied Biosystems (7900 HT, Thermo Fisher Scientific), with the exception that an extension temperature of $61^{\circ} \mathrm{C}$ was used in the assay to measure ChREBP $\beta$.

\section{Western blots}

Cells from three donors treated with $10 \mu \mathrm{M}$ naringenin for 6 days or untreated controls were lysed in radioimmunoprecipitation assay buffer containing a mixture of protease and phosphatase. TGX sodium dodecyl sulfate-polyacrylamide gel electrophoresis (SDS-PAGE) gels (Any $\mathrm{kD}$, Bio-Rad) were used to separate $50 \mu \mathrm{g}$ of solubilized protein per sample. Following transfer, nitrocellulose membranes were probed overnight at $4^{\circ} \mathrm{C}$ with primary antibodies against UCP1 (\#MAB6158, R\&D Systems), GLUT4 (\#sc-53566, Santa Cruz), ChREBP (\#sc515922, Santa Cruz), AMPK (\#2535S, CST), phosphorylated AMPK (T-172, \#2532S, CST), and $\beta$-actin (A5316, Sigma). Horseradish peroxidaselinked anti-rabbit (12-348, Sigma) and anti-mouse (AP130P, Sigma) antibodies were used to detect specific antibody-antigen complexes. Proteins were visualized by chemiluminescence (Western Lightning Plus-ECL, PerkinElmer, Waltham, Massachusetts). Densitometric analyses were performed using Image J software (National Institutes of Health), and the relative expression of the target protein versus $\beta$-actin was calculated. 


\section{Mitochondrial bioenergetics}

To study mitochondrial bioenergetics in human white adipocytes, a Seahorse XF24 Extracellular Flux Analyzer (Agilent) was used. The human adipose-derived stem cells were seeded onto the Seahorse XF24 V7 plate. The protocol for evaluating the bioenergetics parameters of the adipocytes was previously described (20). Briefly, sequential injections of oligomycin $(2 \mu \mathrm{M})$ to inhibit ATP synthase, FCCP $(2 \mu \mathrm{M})$ to uncouple electron transport and allow for maximum electron flux through the electron transport chain, and antimycin A $(1.5 \mu \mathrm{g} / \mathrm{mL})$ to inhibit complex III were used to determine oxygen consumption rate (OCR). Basal OCR was measured before any injections. The difference between basal OCR and respiration that resulted when oligomycin was injected reflects ATP-linked OCR. Following FCCP injection, maximal OCR was measured, and the difference between this value and basal OCR reflects the reserve capacity. Nonmitochondrial OCR was measured after injecting antimycin $\mathrm{A}$, and the difference between this value and OCR that resulted from inhibition of ATP synthase by oligomycin was the basal proton leak.

Cells were treated every other day over 5 days with $8 \mu \mathrm{M}$ naringenin, $0.1 \mu \mathrm{M}$ rosiglitazone, or $0.3 \mu \mathrm{M}$ GW 7647 or were untreated controls. Prior to the assay on day $6,625 \mu \mathrm{L}$ per well serum-free Seahorse $\mathrm{XF}$ assay media, containing $5 \mathrm{mM}$ pyruvate, was loaded onto the plates. Plates were equilibrated in a non- $\mathrm{CO}_{2}$ incubator at $37^{\circ} \mathrm{C}$ for 20 minutes before obtaining the bioenergetics profiles. Experiments included four plates (4-8 replicates) with naringenin, rosiglitazone, and control as the treatments, and two plates also included GW 7647 as a treatment.

\section{Statistical analysis}

A general linear model was used to perform analysis of variance (ANOVA). The primary outcomes, which were differences from the controls, were analyzed after Welch test of homogeneity of variances. The assumption of normality was assessed using the ShapiroWilk test. For data that were not normally distributed, statistical significance was verified by logarithm or square root transformations or by nonparametric analysis (Wilcoxon rank sum test). For the
Seahorse data, a general linear model was used to perform ANOVA to assess the statistical significance of differences among the four treatments (naringenin, rosiglitazone, GW 7647, and control) after accounting for statistical variability in the respective outcomes that was attributable to plates. Six separate models were analyzed with each of the six outcomes (basal, ATP-linked, maximal, reserve, proton-linked, and nonmitochondrial OCR) taken in turn as the dependent variable and as a linear function of models containing the explanatory variables (treatment, plates nested within treatments, and wells nested within plates). The magnitude of the component of outcome variance attributable to plates was tested against the alternative of being significantly different from zero, and if there was strong evidence in favor of the alternative, the mean square for plates within treatments was used as a denominator in testing for treatment differences. If there was not strong evidence in favor of a significant component of variability attributable to plates, the mean square for wells within plates was used as a denominator in testing for treatment differences. Statistical significance of specific pairwise differences between treatments was tested by formulating specific contrasts among the four treatments. Significance was set at $P<0.05$. Outcomes are summarized as mean \pm SE. All analyses were performed using SAS 9.4 (SAS Institute, Cary, North Carolina).

\section{Results \\ Naringenin promotes thermogenic gene expression in human adipocytes}

As shown in Figure 1, naringenin treatment increased mRNA levels of UCP1, peroxisome proliferator-activated receptor gamma (PPAR $\gamma$ ) coactivator (PGC) 1 alpha (PGC-1 $\alpha$, also known as PPARGC1A), and (PPAR $\gamma$ ) coactivator 1 beta (PGC-1 $\beta$, also known as PPARGC1B). PGCs can mediate PPAR $\gamma$-dependent transcriptional responses and are involved in mitochondrial biogenesis and uncoupling. PGC- $1 \alpha$ and PGC-1 $\beta$ can exhibit different regulation patterns (21). Although the induction of PGC- $1 \alpha$ was small (40\% over controls), the regulation was statistically significant $(P=0.013)$.

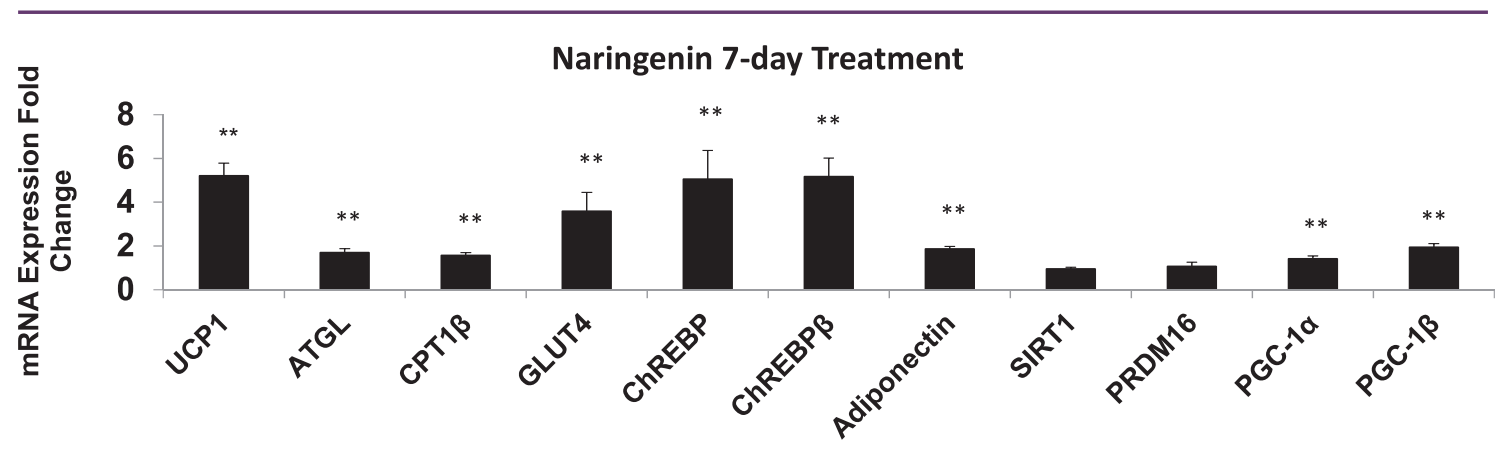

Figure 1 Quantitative real-time polymerase chain reaction (qRT-PCR) in human adipocytes treated with naringenin. qRT-PCR assays for mRNA expression conducted in duplicates following naringenin treatment of hADSC for 7 days compared with untreated hADSC (control): uncoupling protein 1 (UCP1, $n=26$ ), adipose triglyceride lipase (ATGL, $n=12$ ), carnitine palmitoyltransferase 1B (CPT1B, $n=16$ ), glucose transporter type 4 (GLUT4, $n=16$ ), carbohydrate-responsive element-binding protein $\alpha+\beta$ (ChREBP, $n=12$ ), ChREBP beta isoform (ChREBP $\beta, n=12)$, adiponectin $(n=12)$, sirtuin 1 (SIRT1, $n=4)$, PR domain containing 16 (PRDM16, $n=12$ ), and peroxisome proliferatoractivated receptor gamma coactivator 1 alpha/beta $(\mathrm{PGC}-1 \alpha / \beta, n=12)$. Results are presented as mean \pm SE. ${ }^{* *} P<0.01$, compared with respective controls. Each experiment was conducted with four samples except for measurement of UCP1 mRNA that had four or six samples. 


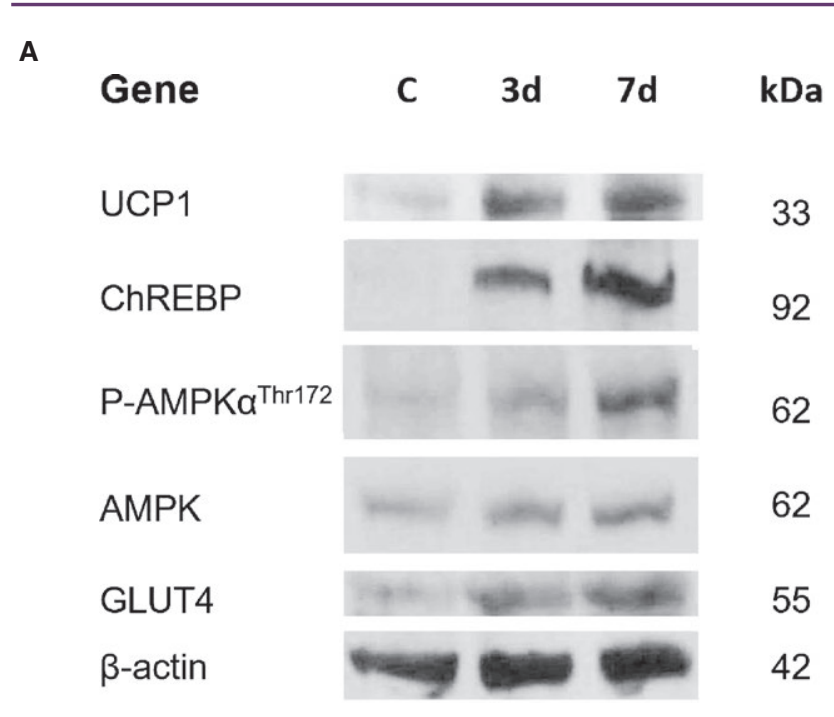

B

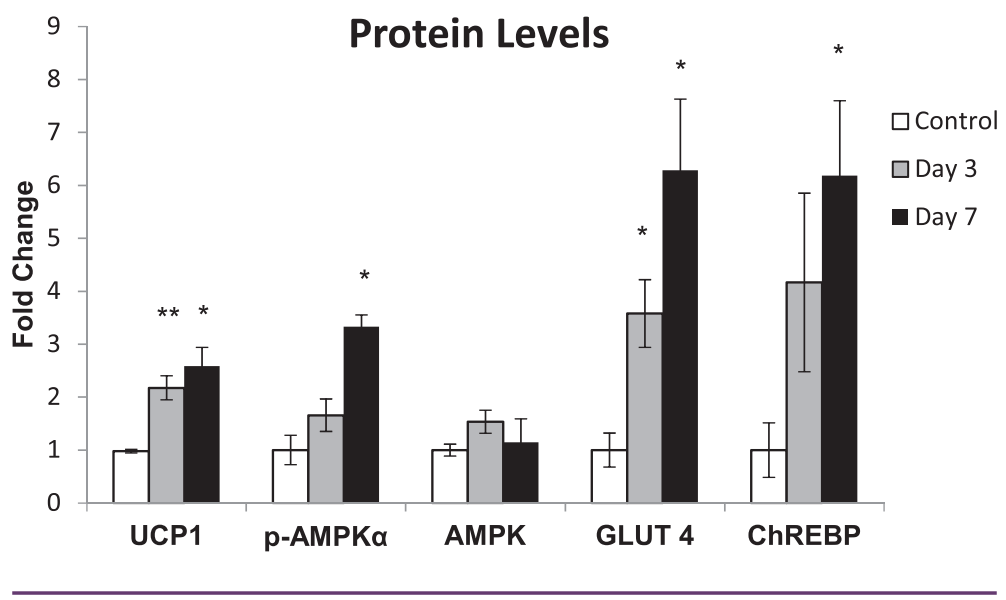

Figure 2 Immunoblotting in human adipocytes treated with naringenin. Human adipocytes from three donors were treated with naringenin for 3 or 7 days. (A) Cellular protein was extracted, and Western blot analysis was used to detect protein levels of uncoupling protein 1 (UCP1), glucose transporter type 4 (GLUT4), and carbohydrate-responsive element-binding protein (ChREBP) and phosphorylation levels of AMP-activated protein kinase (AMPK) in the untreated controls (C) after 3 days (3d) and 7 days (7d) of naringenin treatment. (B) Densitometric analysis. ${ }^{* *} P<0.01,{ }^{\star} P<0.05$.

Fat mobilization in adipose tissue is effected by adipose triglyceride lipase (ATGL) (22). We observed that ATGL (also known as patatin like phospholipase domain containing 2 [PNPLA2]) mRNA increased with naringenin treatment. Expression of the gene encoding carnitine palmitoyltransferase $1 \mathrm{~B}$, which transports fatty acids into the mitochondria, was increased by $55 \%$ over controls, but this regulation was statistically significant $(P<0.01)$. The mRNA expression of genes associated with insulin sensitivity, GLUT4, ChREBP (also known as MLX interacting protein like [MLXIPL]) (which reflects $\alpha+\beta$ ), ChREBP $\beta$, and adiponectin, increased in the naringenin-treated adipocytes $(P<0.01)$. Although sirtuin 1 activity increases the thermogenic capacity of white adipose tissue by mediating PR domain containing 16-driven activation of thermogenesis (23), we did not observe any changes in the mRNA expression of sirtuin 1 or PR domain containing 16 (Figure 1).
Chronic AMPK activation is involved in the remodeling of adipose tissue through upregulation of pathways that favor energy dissipation (24). In hADSC, naringenin treatment increased protein expression of UCP1 (3-day: $P<0.01,7$-day: $P=0.01$ ), GLUT4 (3-day and 7-day: $P=0.02$ ), and ChREBP (7-day: $P=0.02$ ) and phosphorylation of AMPK (7-day: $P=0.02$ ) (Figure 2).

\section{In primary adipose tissue, response to naringenin varies among subjects}

To more closely model the response of adult white adipose tissue in vivo, pWAT was maintained in organ culture with exposure to vehicle or naringenin for 7 or 14 days. There were marked differences in the response to naringenin treatment in the adipose 


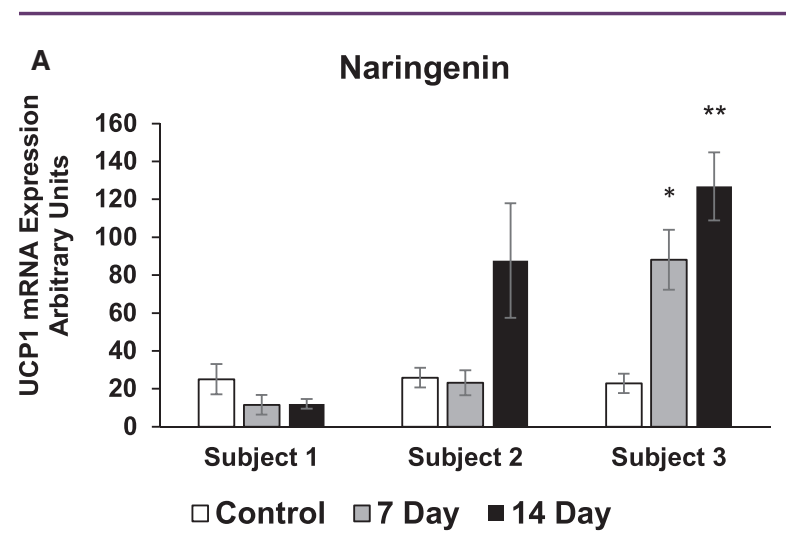

B Naringenin

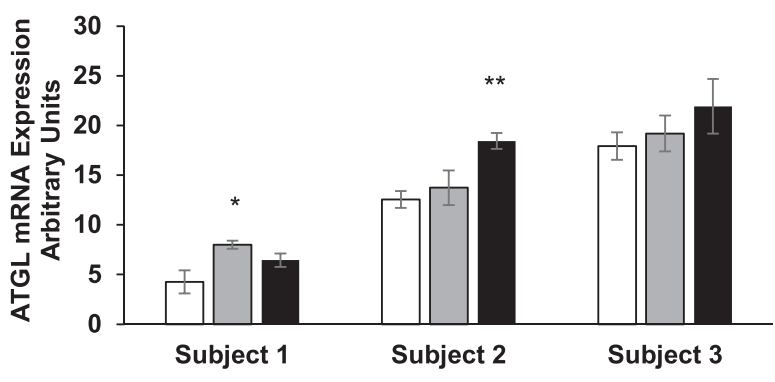

$\square$ Control $\square 7$ Day $\square 14$ Day

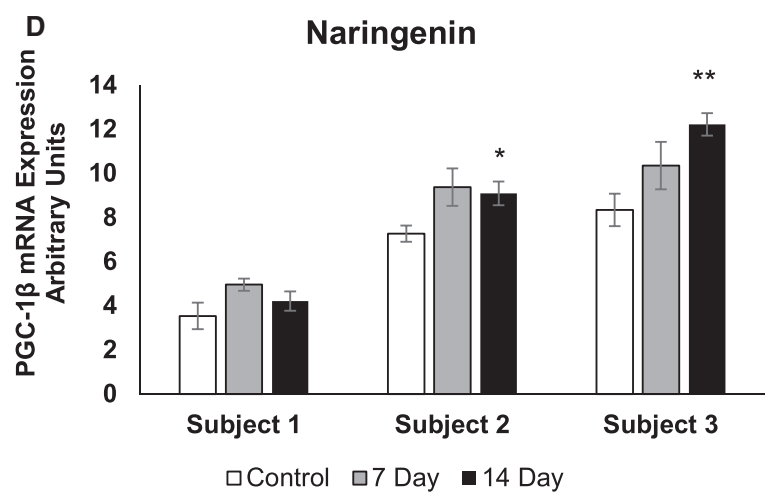

$\mathbf{F}$
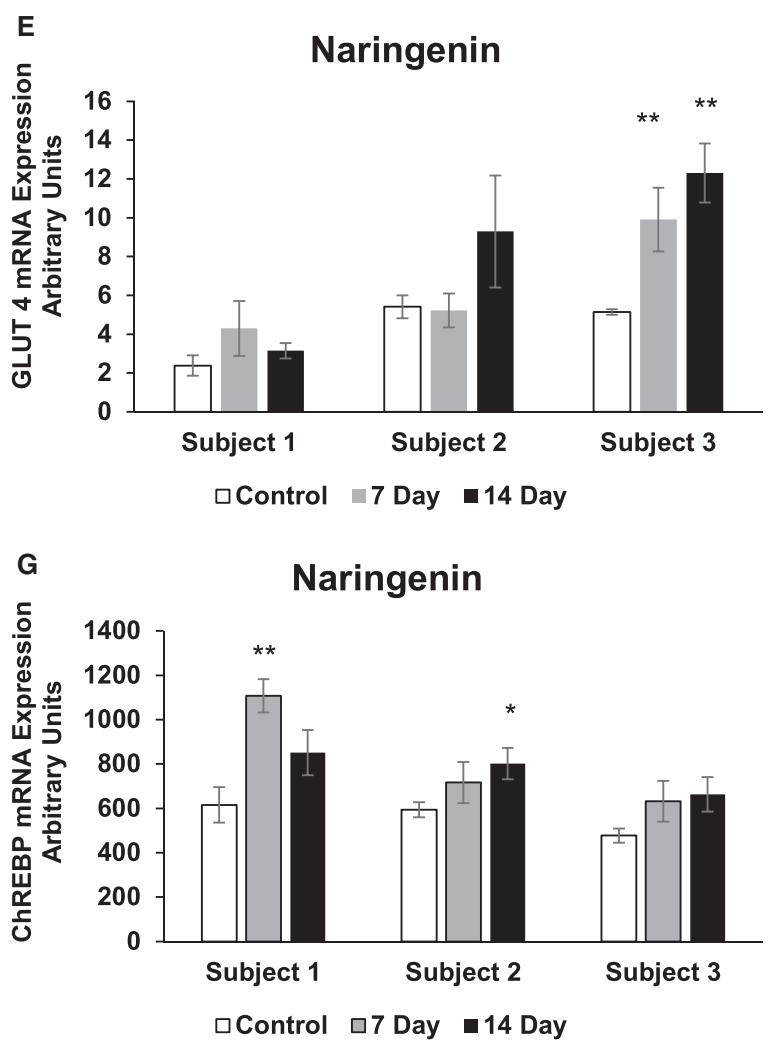
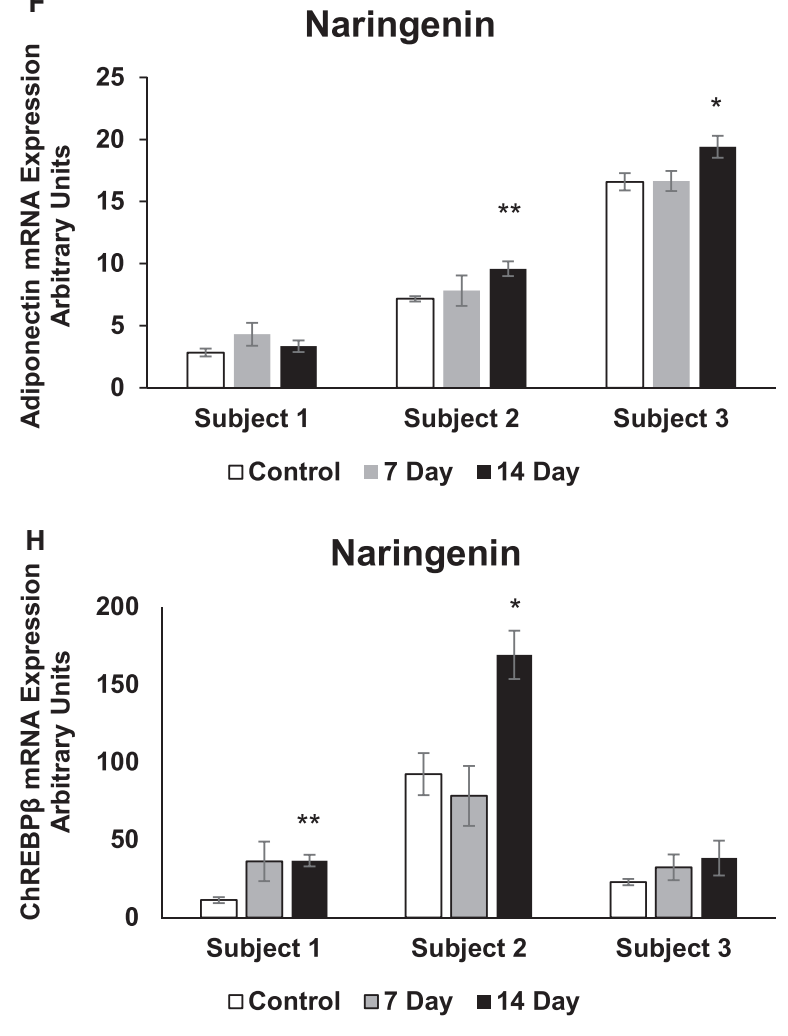

Figure 3 Quantitative real-time polymerase chain reaction (qRT-PCR) in primary human adipose tissue treated with naringenin. qRT-PCR assays for mRNA expression conducted in duplicates following naringenin treatment of quadruplicate pWAT samples, from three female donors with overweight, for 7 and 14 days compared with untreated pWAT (control): (A) uncoupling protein 1 (UCP1), (B) adipose triglyceride lipase (ATGL), (C) peroxisome proliferator-activated receptor gamma coactivator 1 alpha (PGC-1 $\alpha$ ), (D) peroxisome proliferator-activated receptor gamma coactivator 1 beta (PGC-1 $\beta$ ), (E) glucose transporter type 4 (GLUT4), (F) adiponectin, (G) carbohydrate-responsive element-binding protein $\alpha+\beta(C h R E B P)$, and (H) ChREBP beta isoform $(\mathrm{ChREBP} \beta)$. Results are presented as mean \pm SE. ${ }^{\star \star} P<0.01$, ${ }^{\star} P<0.05$, compared with respective controls. 
tissues from the three different subjects. In subject 1, mRNA expression of UCP1 did not increase. In subject 2, there was a substantial increase in UCP1, but the variability among the samples precluded attainment of statistical significance. However, in subject 3 , the increase in UCP1 expression was statistically significant at both 7 and 14 days $(P=0.008$ and $P=0.001$, respectively) of naringenin treatment. The expression of GLUT4 followed a similar pattern $(P=0.03$ and $P=0.003$ at 7 and 14 days, respectively). Although ChREBP is expressed in brown adipose tissue, induction of UCP1 was not accompanied by increased expression of ChREBP $\beta$ in subject 3. ChREBP $\beta$ expression increased in subjects 1 and $2(P=0.002$ and $P=0.013$, respectively) at 14 days of naringenin treatment. In pWAT of subjects 1 and 3, PGC- $1 \alpha$ increased at days 7 and 14 , respectively $(P=0.04$ and $P=0.02)$, whereas PGC-1 $\beta$ increased at 14 days in subjects 2 and $3(P=0.03$ and $P=0.005$, respectively). In pWAT of subjects 1 and 2 , ATGL increased at days 7 and 14 , respectively $(P=0.02$ and $P=0.003)$, and adiponectin increased at 14 days in pWAT of subjects 2 and 3 ( $P=0.008$ and $P=0.005$, respectively) (Figure 3 ).

In pWAT from three subjects (including subjects 1,2 , and 4), we examined the mRNA expression of UCP1 and GLUT4 following treatment with the pharmacologic PPAR $\alpha$ agonist GW 7647. Similar to the naringenin treatment, in pWAT from subject 1 , there was no increase in the mRNA expression of UCP1 and GLUT4, whereas in pWAT from subject 2, there was a significant increase in UCP1 ( $P=0.003$ and $P=0.004$ at 7 and 14 days, respectively) and a substantial increase in GLUT4, but this regulation was not statistically significant (Figure 4).

\section{Naringenin increases energy expenditure}

As shown in Figure 5, naringenin increased basal, ATP-linked, maximal, and reserve OCR in intact adipocytes compared with controls $(P<0.01)$. Basal proton-linked OCR and nonmitochondrial OCR were not significantly different between vehicle and treatment groups. ATPlinked, maximal, reserve, and proton-linked respiration in human adipocytes exposed to naringenin was similar to those of the positive controls, rosiglitazone, and GW $7647(P>0.05)$.

\section{Discussion}

Pharmacologic browning of fat correlates with resistance to high-fat diet-induced obesity and insulin resistance. In our study, we demonstrated an increase in the mRNA and protein expression of UCP1, GLUT4, and ChREBP, the genes involved in thermogenesis and insulin sensitivity, when hADSC were treated with naringenin at concentrations that are physiologically attainable in humans (18). Naringenin also stimulated mRNA expression of PGC- $1 \alpha$ and PGC-1 $\beta$ (nuclear receptor coactivators involved in thermogenesis), ATGL and carnitine palmitoyltransferase 1B (key enzymes necessary for fat oxidation), and adiponectin (insulin-sensitizing adipokine), in addition to increasing OCR.

In pWAT maintained ex vivo, the response was varied among the adipose tissue obtained from three female subjects with overweight. UCP1 and GLUT4 expression significantly increased in one of the three subjects, which was accompanied by an increase in the expression of PGC- $1 \alpha$ and PGC- $1 \beta$. However, expression of ChREBP and ChREBP $\beta$ increased in pWAT of the subjects who did not manifest an increase in UCP1 and GLUT4. Interestingly, the lack of any change in UCP1 and GLUT4 expression in response to naringenin treatment in pWAT of subject 1 was also seen when this pWAT was treated with GW 7647, a potent pharmacologic PPAR $\alpha$ agonist. In human adipocytes, PPAR $\alpha$ has been shown to upregulate the expression of the genes involved in lipid metabolism and increase maximal OCR (25). Because naringenin activates PPAR $\alpha(10)$, it is likely that PPAR $\alpha$ plays an important role in the thermogenic effect of naringenin, but some humans may not be responders to this pathway. However, the notion bears investigation with larger sample sizes.

Browning of human adipocytes is induced by pharmacologic PPAR $\alpha$ and $\gamma$ agonists, GW 7647 and rosiglitazone, respectively, through activation of a comprehensive gene program that leads to increased mitochondrial function and energy consumption $(19,26,27)$. Naringenin is an activator of PPAR $\alpha$ and $\gamma$ (10). In mice, naringenin has been shown to increase energy expenditure, upregulate UCP1 in brown fat, and promote the induction of fat oxidation $(12,28)$. Our in vitro studies in human adipocytes suggest that naringenin may induce a browning of

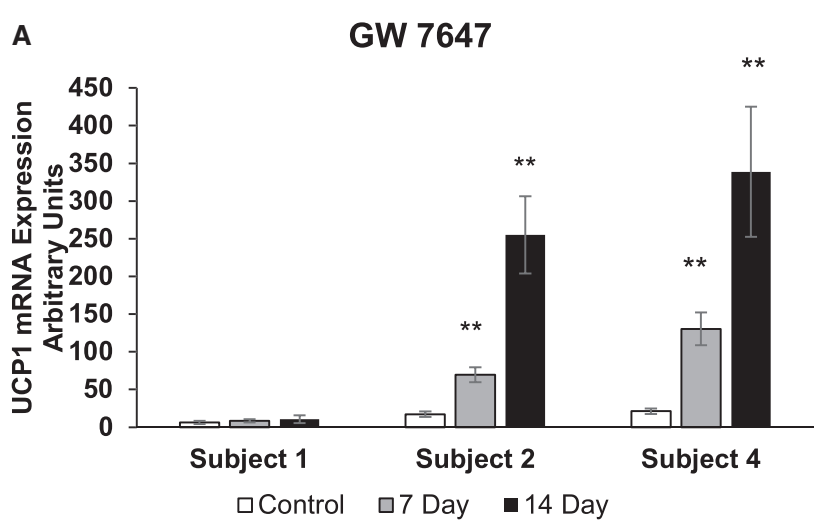

B

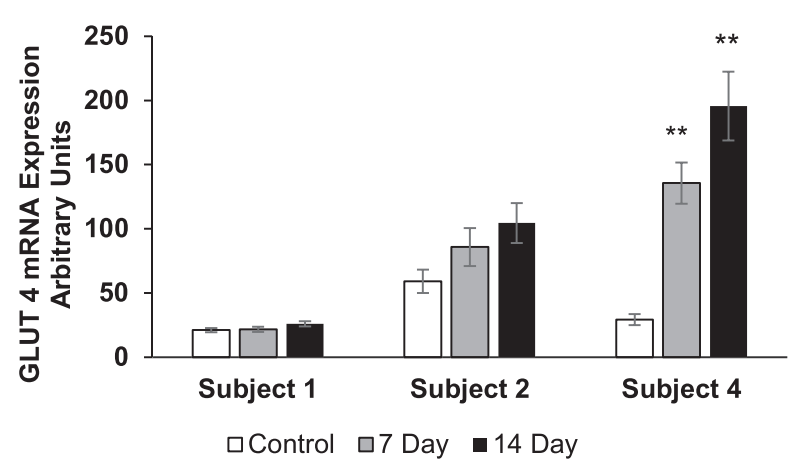

Figure 4 Quantitative real-time polymerase chain reaction (qRT-PCR) in primary human adipose tissue treated with GW 7647. qRT-PCR assays for mRNA expression conducted in duplicates following GW 7647 (pharmacologic peroxisome proliferator-activated receptor alpha agonist) treatment of quadruplicate pWAT samples, from three female donors with overweight, for 7 and 14 days compared with untreated pWAT (control): (A) uncoupling protein 1 (UCP1) and (B) glucose transporter type 4 (GLUT4). Results are presented as mean \pm SE. ${ }^{\star \star} P<0.01$, compared with respective controls. 


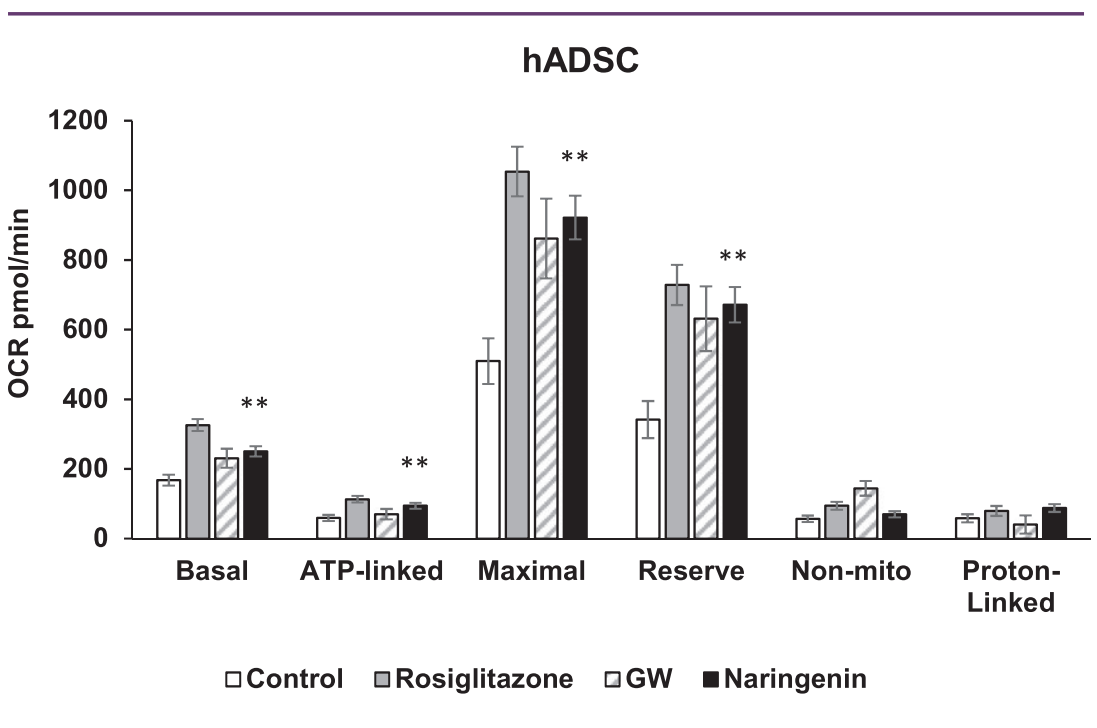

Figure $\mathbf{5}$ Oxygen consumption rate (OCR) in human adipocytes treated with naringenin. OCR was measured in mature human adipocytes that were grown in four Seahorse XF24 V7 plates. Cells were treated for 6 days with naringenin, rosiglitazone, or GW 7647 or were untreated controls. Measurements include basal OCR, ATP-linked OCR after inhibition of ATP synthase by oligomycin, maximal respiratory rate, and reserve capacity after artificial uncoupling by carbonyl cyanide-4(trifluoromethoxy) phenylhydrazone; nonmitochondrial respiration after inhibition of complex III by antimycin A; and proton-linked OCR. Basal, ATP-linked, maximal, and reserve OCR were significantly different between the naringenin-treated cells and the untreated controls. ATP-linked, maximal, reserve, and proton-linked OCR were not significantly different $(P>0.05)$ between naringenin and the positive controls rosiglitazone and GW 7647. Results are presented as mean \pm SE. ${ }^{* *} P<0.01$, compared with control.

human white adipose tissue through an upregulation of UCP1 (Figures 1 and 2) and an increase in OCR (Figure 5). Additionally, the expression of the PPAR $\gamma$ coactivators PGC- $1 \alpha$ and $\beta$ was stimulated in human adipocytes and pWAT. Both members of the PGC1 family are important for mitochondrial oxidative metabolism, although differences in their interactions with other transcription factors mediate differences in their functional specificity (29).

The relevance of increased energy consumption induced by browning to human physiology may be debatable, but its effects on glucose metabolism have physiologic significance. In humans, approximately $70 \mathrm{~mL}$ of brown fat can clear 20 to $25 \mathrm{~g}$ of glucose from circulation in 24 hours (30). Our in vitro studies in human adipocytes clearly demonstrate that naringenin increases expression of ChREBP, ChREBP $\beta$, and GLUT4. In white adipose tissue, the transcriptional targets of ChREBP include the genes involved in adipocyte differentiation, lipid metabolism, and browning (6). Mice with adipose-specific GLUT4 overexpression have displayed improved glucose control and upregulation of ChREBP expression (4). In humans grouped according to their 2-hour blood glucose concentrations, the expression of GLUT4 and ChREBP measured in subcutaneous fat biopsies decreased as blood glucose concentrations rose across the groups (5). Interestingly, expression of adipose ChREBP correlated positively with expression of GLUT4 and insulin sensitivity (5). Although the study was done in adolescents with obesity, it provides preliminary evidence that in humans, ChREBP, ChREBP $\beta$, and GLUT4 gene expression correlates with physiologic outcomes related to glucose homeostasis. Secretion of adipokines such as adiponectin is another key role of adipose tissue that contributes to whole-body insulin sensitivity (31). Thiazolidinediones are PPAR $\gamma$ agonists that increase insulin sensitivity at least in part through an increase in adiponectin secretion (32). In the promoter region of the human adiponectin gene, a PPAR-responsive element has been identified and is thought to be the mechanism by which thiazolidinediones increase plasma adiponectin (33). In our study, naringenin treatment increased adiponectin mRNA expression, suggesting a role for naringenin in protecting against insulin resistance.

Shortage of cellular energy stores marked by an increase in AMP levels and a decline in ATP levels activates AMPK, which then initiates cellular programs that increase catabolism, mostly by enhancing oxidative metabolism and mitochondrial biogenesis (34). Activation of AMPK increases UCP1 and induces an accumulation of brown adipocytes in murine white adipose tissue (35). Naringenin promotes glucose homeostasis in animal models through activation of AMPK signaling in the liver, skeletal muscle, and macrophages differentiated from the human cell line THP-1 (36). In our study, activation of AMPK was stimulated in human adipocytes treated with naringenin (Figure 2). Consistent with the activation of AMPK and increase in catabolism, our studies show that naringenin increases mRNA expression of ATGL in hADSC and pWAT. Catabolism of fatty acids from triglyceride stores is the function of lipolytic enzymes, particularly ATGL, which catalyzes the initial step in triglyceride hydrolysis (22).

The distinctive function of brown adipocytes is thermogenic energy expenditure, propelled by the catabolic breakdown of lipids. 
Therefore, to distinguish between white adipocytes and those that have potentially acquired a brown/beige phenotype, we extended our characterization to the mitochondrial function of naringenin-treated adipocytes by analyzing OCR. Basal OCR and ATP-linked OCR were higher in the naringenin-treated cells compared with the control or untreated cells. To functionally determine browning in hADSC, higher substrate oxidation capacity than controls must be demonstrated as would be reflected by substantial increases in maximal respiration rate. Following sequential injection of compounds that modulate mitochondrial function, naringenin increased maximal respiratory capacity, and this increase was not different from that of the positive controls rosiglitazone and GW 7647. However, induction of UCP1 following treatment with naringenin is by far lower than UCP1 induction by rosiglitazone in the adipocytes (37). Therefore, the increase in energy expenditure may not be completely mediated by UCP1. Proton leak was not significantly different between naringenin and controls. UCP1 is not inherently active in the mitochondria and is activated by fatty acids. In intact cells, protonleakrepresents basaluncoupledrespiration.Inrodentadipocytes, injecting isoproterenol during the OCR measurement stimulates an adrenergic response, and UCP1-mediated proton leak can be determined (38). However, in human adipocytes from individuals with obesity, the response to $\beta$-adrenergic stimulation by isoproterenol is impaired (39).

\section{Conclusion}

Robust data from rodent studies and in vitro models demonstrate that naringenin increases energy expenditure and improves glucose metabolism. Although our study in hADSC and pWAT builds upon these data, it demonstrates that the response to naringenin may differ among human subjects based on their response to pathways that activate energy expenditure. The results warrant further investigation to determine their clinical relevance. 0

\section{(C) 2018 The Obesity Society}

\section{References}

1. Harms M, Seale P. Brown and beige fat: development, function and therapeutic potential. Nat Med 2013;19:1252-1263.

2. Ouellet V, Routhier-Labadie A, Bellemare W, et al. Outdoor temperature, age, sex, body mass index, and diabetic status determine the prevalence, mass, and glucose-uptake activity of 18F-FDG-detected BAT in humans. J Clin Endocrinol Metab 2011;96:192-199.

3. Eissing L, Scherer T, Todter K, et al. De novo lipogenesis in human fat and liver is linked to ChREBP-beta and metabolic health. Nat Commun 2013;4:1528. doi:10.1038/ ncomms 2537

4. Herman MA, Peroni OD, Villoria J, et al. A novel ChREBP isoform in adipose tissue regulates systemic glucose metabolism. Nature 2012;484:333-338.

5. Kursawe R, Caprio S, Giannini C, et al. Decreased transcription of ChREBP-alpha/ beta isoforms in abdominal subcutaneous adipose tissue of obese adolescents with prediabetes or early type 2 diabetes: associations with insulin resistance and hyperglycemia. Diabetes 2013;62:837-844.

6. Nuotio-Antar AM, Poungvarin N, Li M, et al. FABP4-Cre mediated expression of constitutively active ChREBP protects against obesity, fatty liver, and insulin resistance. Endocrinology 2015;156:4020-4032.

7. Decara J, Arrabal S, Beiroa D, et al. Antiobesity efficacy of GLP-1 receptor agonist liraglutide is associated with peripheral tissue-specific modulation of lipid metabolic regulators. Biofactors 2016;42:600-611.

8. Zygmunt K, Faubert B, MacNeil J, Tsiani E. Naringenin, a citrus flavonoid, increases muscle cell glucose uptake via AMPK. Biochem Biophys Res Comm 2010;398:178-183.

9. Kannappan S, Anuradha CV. Naringenin enhances insulin-stimulated tyrosine phosphorylation and improves the cellular actions of insulin in a dietary model of metabolic syndrome. Eur J Nutr 2010;49:101-109.
10. Goldwasser J, Cohen PY, Yang E, Balaguer P, Yarmush ML, Nahmias Y. Transcriptional regulation of human and rat hepatic lipid metabolism by the grapefruit flavonoid naringenin: role of PPARalpha, PPARgamma and LXRalpha. PLoS One 2010;5:e12399. doi:10.1371/journal.pone.0012399

11. Bhattacharya S, Oksbjerg N, Young JF, Jeppesen PB. Caffeic acid, naringenin and quercetin enhance glucose-stimulated insulin secretion and glucose sensitivity in INS-1E cells. Diabetes Obes Metab 2014;16:602-612.

12. Thaiss CA, Itav S, Rothschild D, et al. Persistent microbiome alterations modulate the rate of post-dieting weight regain. Nature 2016;540:544-551.

13. Mulvihill EE, Burke AC, Huff MW. Citrus flavonoids as regulators of lipoprotein metabolism and atherosclerosis. Annu Rev Nutr 2016;36:275-299.

14. Kiani J, Imam SZ. Medicinal importance of grapefruit juice and its interaction with various drugs. Nutr J 2007;6:33. doi:10.1186/1475-2891-6-33

15. Edwards DJ, Bernier SM. Naringin and naringenin are not the primary CYP3A inhibitors in grapefruit juice. Life Sci 1996;59:1025-1030.

16. Bailey DG, Kreeft JH, Munoz C, Freeman DJ, Bend JR. Grapefruit juice-felodipine interaction: effect of naringin and $6^{\prime}, 7^{\prime}$-dihydroxybergamottin in humans. Clin Pharmacol Ther 1998;64:248-256.

17. Ho PC, Saville DJ, Wanwimolruk S. Inhibition of human CYP3A4 activity by grapefruit flavonoids, furanocoumarins and related compounds. J Pharm Pharm Sci 2001:4:217-227.

18. Kanaze FI, Bounartzi MI, Georgarakis M, Niopas I. Pharmacokinetics of the citrus flavanone aglycones hesperetin and naringenin after single oral administration in human subjects. Eur J Clin Nutr 2007;61:472-477.

19. Elabd C, Chiellini C, Carmona M, et al. Human multipotent adipose-derived stem cells differentiate into functional brown adipocytes. Stem Cells 2009;27:2753-2760.

20. Dranka BP, Benavides GA, Diers AR, et al. Assessing bioenergetic function in response to oxidative stress by metabolic profiling. Free Radic Biol Med 2011;51:1621-1635.

21. Deng T, Sieglaff DH, Zhang A, et al. A peroxisome proliferator-activated receptor gamma (PPARgamma)/PPARgamma coactivator lbeta autoregulatory loop in adipocyte mitochondrial function. J Biol Chem 2011;286:30723-30731.

22. Zimmermann R, Strauss JG, Haemmerle G, et al. Fat mobilization in adipose tissue is promoted by adipose triglyceride lipase. Science 2004;306:1383-1386.

23. Jokinen R, Pirnes-Karhu S, Pietilainen KH, Pirinen E. Adipose tissue NAD(+)homeostasis, sirtuins and poly(ADP-ribose) polymerases -important players in mitochondrial metabolism and metabolic health. Redox Biol 2017;12:246-263.

24. Vila-Bedmar R, Lorenzo M, Fernandez-Veledo S. Adenosine 5'-monophosphate-activated protein kinase-mammalian target of rapamycin cross talk regulates brown adipocyte differentiation. Endocrinology 2010;151:980-992.

25. Barquissau V, Beuzelin D, Pisani DF, et al. White-to-brite conversion in human adipocytes promotes metabolic reprogramming towards fatty acid anabolic and catabolic pathways. Mol Metab 2016;5:352-365.

26. Pisani DF, Djedaini M, Beranger GE, et al. Differentiation of human adipose-derived stem cells into "brite" (brown-in-white) adipocytes. Front Endocrinol (Lausanne) 2011;2:87. doi:10.3389/fendo.2011.00087

27. Loft A, Forss I, Siersbaek MS, et al. Browning of human adipocytes requires KLF1 and reprogramming of PPARgamma superenhancers. Genes Dev 2015;29:7-22.

28. Mulvihill EE, Allister EM, Sutherland BG, et al. Naringenin prevents dyslipidemia, apolipoprotein B overproduction, and hyperinsulinemia in LDL receptor-null mice with diet-induced insulin resistance. Diabetes 2009;58:2198-2210.

29. Scarpulla RC. Transcriptional paradigms in mammalian mitochondrial biogenesis and function. Physiol Rev 2008;88:611-638.

30. Chondronikola M, Volpi E, Borsheim E, et al. Brown adipose tissue improves whole-body glucose homeostasis and insulin sensitivity in humans. Diabetes 2014;63:4089-4099.

31. Arioglu E, Rother KI, Reitman ML, Premkumar A, Taylor SI. Lipoatrophy syndromes: when 'too little fat' is a clinical problem. Pediatr Diabetes 2000;1:155-168.

32. Combs TP, Wagner JA, Berger J, et al. Induction of adipocyte complement-related protein of 30 kilodaltons by PPARgamma agonists: a potential mechanism of insulin sensitization. Endocrinology 2002;143:998-1007.

33. Iwaki M, Matsuda M, Maeda N, et al. Induction of adiponectin, a fat-derived antidiabetic and antiatherogenic factor, by nuclear receptors. Diabetes 2003;52:1655-1663

34. Zong H, Ren JM, Young LH, et al. AMP kinase is required for mitochondrial biogenesis in skeletal muscle in response to chronic energy deprivation. Proc Natl Acad Sci U S A 2002;99:15983-15987.

35. Ng M, Fleming T, Robinson M, et al. Global, regional, and national prevalence of overweight and obesity in children and adults during 1980-2013: a systematic analysis for the Global Burden of Disease Study 2013. Lancet 2014;384:766-781.

36. Nyane NA, Tlaila TB, Malefane TG, Ndwandwe DE, Owira PMO. Metformin-like antidiabetic, cardio-protective and non-glycemic effects of naringenin: molecular and pharmacological insights. Eur J Pharmacol 2017;803:103-111.

37. Rebello CJ, Greenway FL, Johnson WD, et al. Fucoxanthin and its metabolite fucoxanthinol do not induce browning in human adipocytes. J Agric Food Chem 2017;65:10915-10924.

38. Li Y, Fromme T, Schweizer S, Schottl T, Klingenspor M. Taking control over intracellular fatty acid levels is essential for the analysis of thermogenic function in cultured primary brown and brite/beige adipocytes. EMBO Rep 2014;15:1069-1076.

39. Yehuda-Shnaidman E, Buehrer B, Pi J, Kumar N, Collins S. Acute stimulation of white adipocyte respiration by PKA-induced lipolysis. Diabetes 2010;59:2474-2483. 